\title{
Mathematics of lymphedema
}

\section{F Passariello ${ }^{1}$}

\author{
${ }^{1}$ Fondazione Vasculab ONLUS, via Francesco Cilea 280 - 80127 Naples, Italy
}

presented to: the VIII theoretical and practical workshop. Diagnosis and treatment of (phlebo)lymphedema. Apr 23rd, 2006, Bologna, Italy submitted: Mar 8, 2017, accepted: Mar 22, 2017, EPub Ahead of Print: Mar 23, 2017, published: May 1, 2017

Conflict of interest: None

DOI: 10.24019/jtavr.19 - Corresponding author: Dr. Fausto Passariello, afunzionale@tiscalinet.it

(C) 2016 Fondazione Vasculab impresa sociale ONLUS. All rights reserved.

Abstract Flow in the extracellular matrix can be the last available resource in advanced lymph dysfunctions. The ultrasound appearance of the subcutaneous tissue in lymph diseases resembles a desert of stones. Flow can be assimilated to the liquid flow through a porous medium. In hydrogeology these phenomena trace back to the Darcy law, by Henry Darcy, French engineer of the XIX century. In a porous medium the area available to flow is generally smaller than the area of the overall cross section and the underlying fluid dynamics occurs in a viscous regimen. The mathematical details of the Darcy representation are here reported. Apart of the general and theoretical interest, these information can be of practical use if they are linked to data coming from the ultrasound investigation of lower limbs.

Keywords Lymphedema, extracellular matrix, Darcy law, porosity, viscous forces, Reynolds number.

\section{Introduction}

The lymphatic circulation sustains side by side the venous drainage in the task of removing tissue catabolites. In details, the lymph system deals with the removal of the remains, non-efficiently taken away by the venous circulation.

The lymph system acts in a much wider temporal scale than the venous system, which is instead able to react in a quicker way to tissue metabolic changes.

The removal of the interstitial fluid occurs essentially by means of two pathways:

The lymph vessels, which originate from the initial lymphatics or pre-lymphatics, which are constituted by an endothelial layer with wide fenestrations; in lymph diseases they have a modified function, for instance cause of an obstruction or cause of their absence (agenesia).

The extracellular matrix (ECM), which can show several considerable modifications, being however always present and constituting the last resource in an advanced lymph disease ${ }^{1}$.

\section{Structure of the extracellular matrix}

Shortly, the ECM is constituted by a structured space, which is placed between the connective cells and contains a colloidal solution, sometimes present as a "sol", sometimes as a "gel". Fibres originating from fibroblasts cross the matrix and link cells each other and to the lymphatic vessels and contribute to make the matrix a structure of constrained elements. Skin handling provokes the fibre strain and can ease or prevent fluid movement (micro-reticulum).

\section{Matrix, porous media and porosity}

The reticular appearance is emphasized in the advanced lymphatic disease, where ECM appears as a reticular material, often detectable in ultrasound images of lymphatic limbs. The sub-cutaneous tissue here is rendered as an image similar to a desert of stones, everywhere fragmented into clamps of several sizes. Hypo-echogenic spaces are placed between clamps of hyper-reflecting material, which is stored in the matrix (macro-reticulum) ${ }^{2}$.

\section{The Darcy Model}

Flow through the matrix occurs in a bed, where several completely impenetrable structures (bones) are present as well as partially penetrable ones (muscles, tendons).

For this reason, ECM flow can be assimilated to the liquid flow through a porous medium. In hydrogeology 
these phenomena trace back to the Darcy law, by Henry Philibert Gaspard Darcy, a French engineer of the XIX century $^{[1] 3-7}$ (Figs. 1-3).

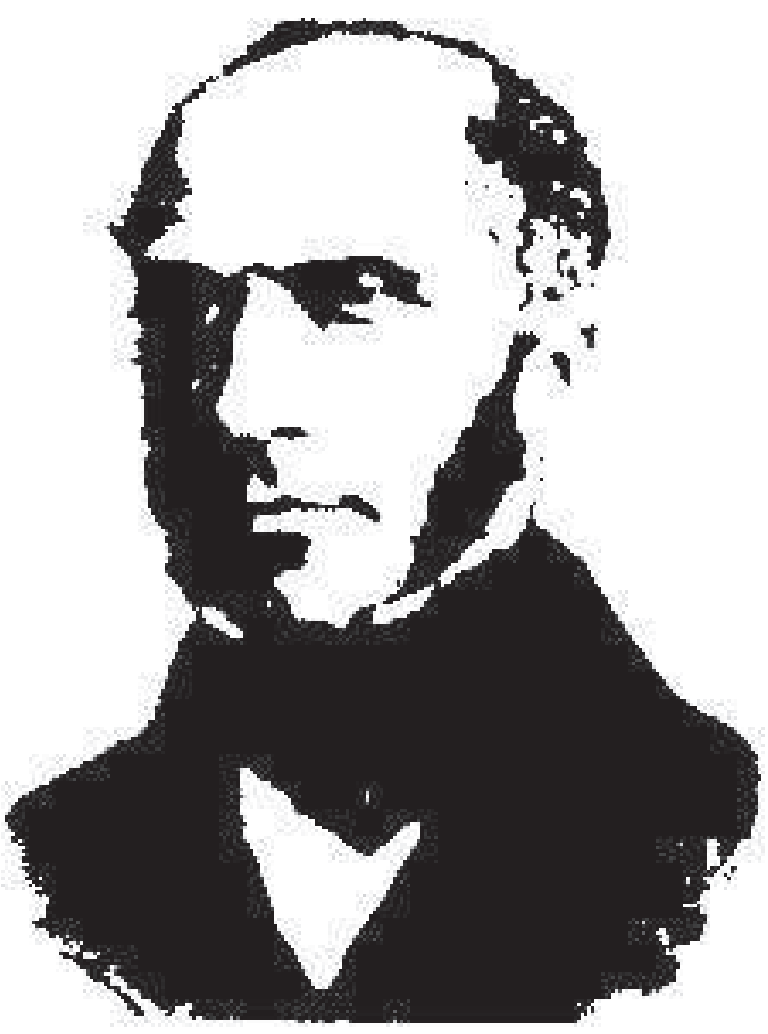

Figure 1 - Henry Philibert Gaspard Darcy. (Dijon 1803 Paris 1858)

\section{Cross section available to flow}

The reticular structure and the aggregation of the interstitial materials allows the similitude between the ECM and a porous medium, i.e. a medium where the fluid penetrates without occupying all the space, but only an available part.

The fluid enters a porous medium in the same way we can afford an obstacle pathway or a walk through the crowd. The space we can use is only the one placed between the obstacles. If someone would measure the crossing velocity, considering just the moment and the point of entry and exit and the distance between these points, he would measure a different velocity than the one experimented by a real traveller, kept busy in avoiding the obstacles encountered on the traveller's pathway.

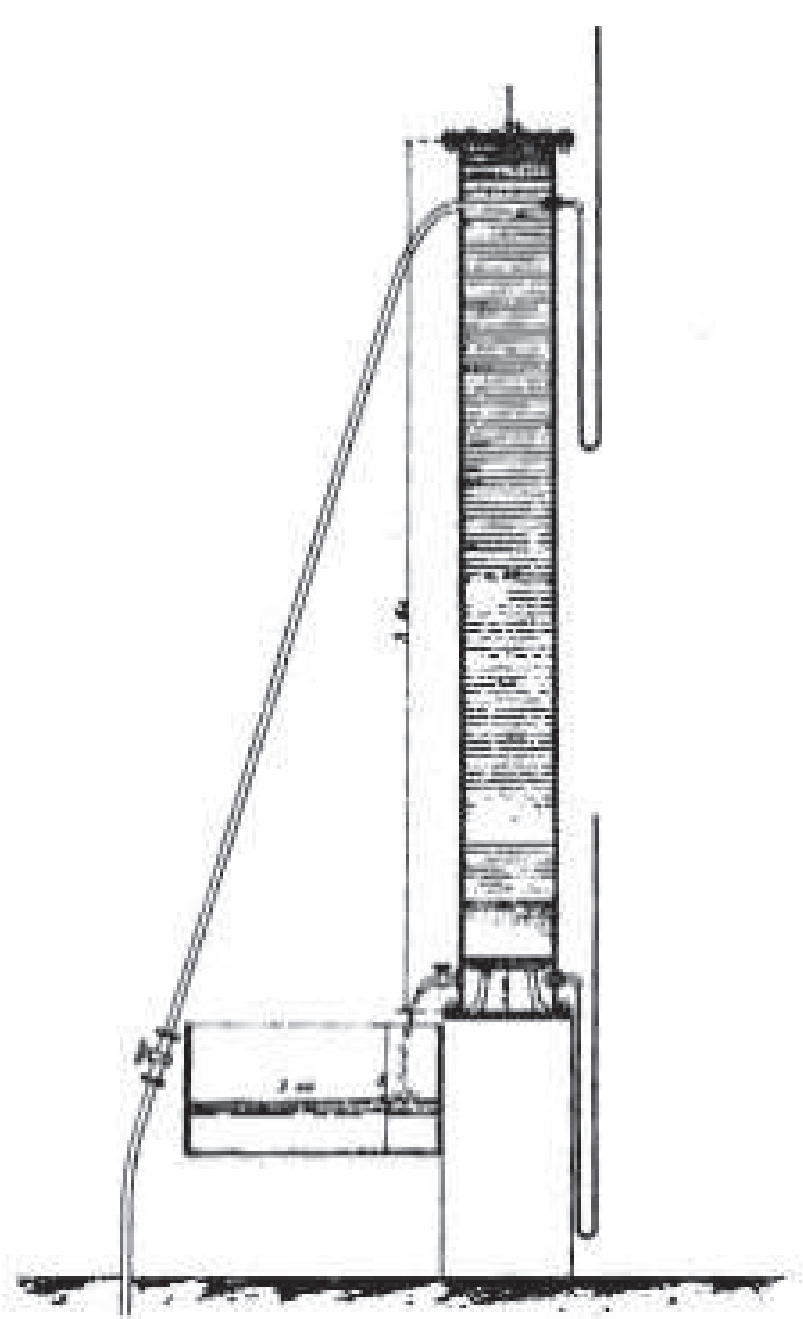

Figure 2 - Apparatus intended to determine the law of the water flow through sand. (Darcy, 1856)

Generally, the pore velocity (vbed) is higher than the upstream or Darcy velocity ( $v_{u p}$ ) and their ratio is the same of the ratio between the upstream area $\left(A_{u p}\right)$ and the porous area $\left(A_{b e d}\right)$ or the area really available to flow. The porosity coefficient $(\mathrm{n})$ can be defined:

$$
\mathrm{n}=\frac{\mathrm{V}_{\mathrm{up}}}{\mathrm{V}_{\mathrm{bed}}}=\frac{\mathrm{A}_{\mathrm{bed}}}{\mathrm{A}_{\mathrm{up}}} ;
$$

where $\mathrm{n}<=1$.

Shortly, the fluid enters the section $\mathrm{A}_{\text {up }}$, but it flows really through a reduced section $A_{b e d}$ with a greater velocity vbed. This is the concept of porosity. 


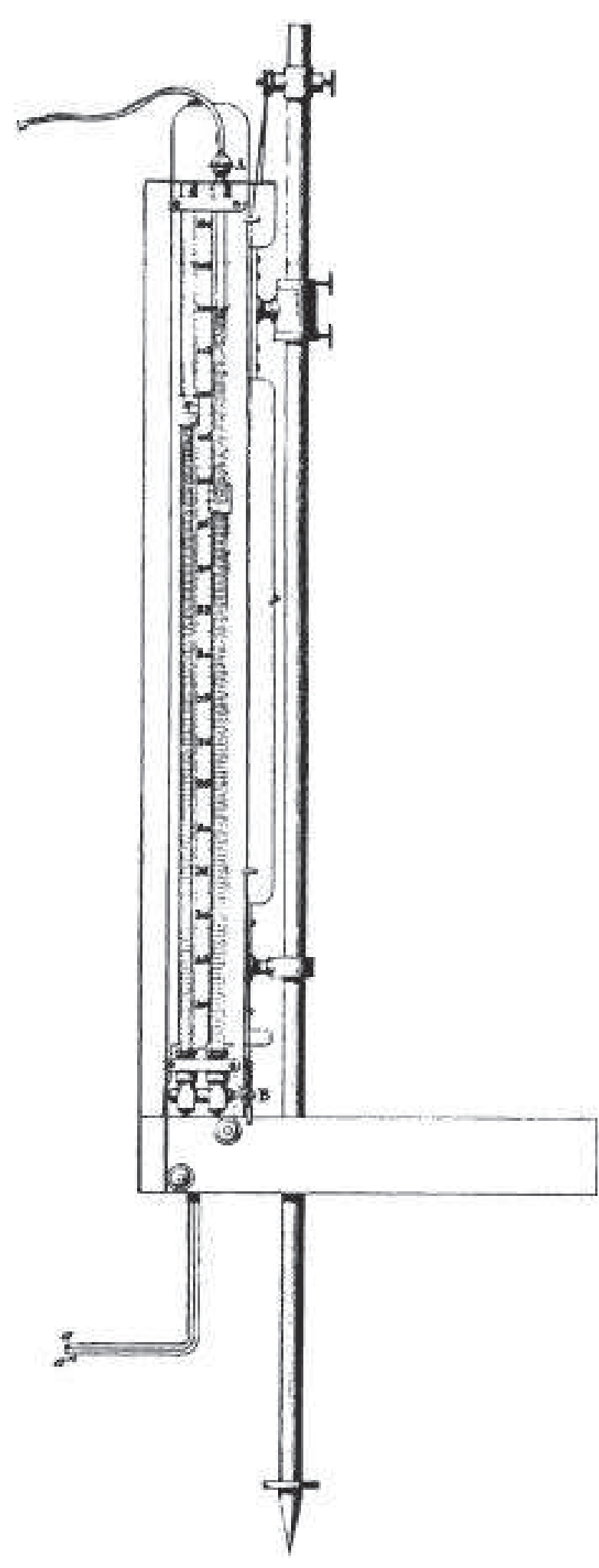

Figure 3 - The Darcy's Pitot tube (1858)

At the ultrasound examination porosity has the appearance of grains of irregular size, placed in layers which are roughly parallel to the skin surface, just about a floor of pebbles. The interstitial fluid worms itself between the layers and sometimes moves them, modelling a stratum of anechoic fluid (lymphatic lake or lymphatic stratum). The grains are the impenetrable or at least partially permeable part and the spaces between the medium grains are the pores. Inside a cross section the area really available to flow is smaller and linearly related to the overall value by the coefficient of porosity.

\section{Active forces in the matrix}

A spherical object, moving in the ECM (as in any other medium), undergoes mainly two different types of forces: the inertial forces, which are needed to accelerate the object, and the viscous forces, which oppose to the movement and slow down it.

Viscous forces $\mathrm{F}_{\mathrm{V}}$ act impeding the sliding of fluid between its contiguous parts and on the obstacles in contact with it.

\section{formula}

Viscous forces can be quantified by the Stokes

$$
\mathrm{F}_{\mathrm{v}}=6 \pi \eta \mathrm{rv}
$$

and depend on the fluid viscosity $\eta$, the spherical radius $\mathbf{r}$ and velocity $\mathbf{v}$.

Inertial forces $\mathrm{F}_{\mathrm{i}}$ can be quantified observing that during its movement the sphere moves the fluid, accelerating it until it reaches its equilibrium velocity. The volume of the moved fluid in $1 \mathrm{~s}$ is included in a cylinder with base area equal to the area of the maximum circle of the sphere and height equal to its velocity, i.e. a volume equal to $\pi r^{2} v$.

Fluid density is $\rho$ and the kinetic energy, which is transferred in $1 \mathrm{~s}$ to the moved fluid is

$$
\frac{1}{2} m v^{2}=\frac{1}{2} \pi r^{2} v \rho v^{2}=\frac{1}{2} \rho \pi r^{2} v^{3}
$$

This energy is equal to the impulse of the inertial forces, i.e. $\mathrm{F}_{\mathrm{i}} \mathrm{v}$.

Matching the kinetic energy in 1s to the impulse, we have:

$$
F_{i}=\frac{1}{2} \rho \pi r^{2} v^{2}
$$

The Reynolds number $\mathrm{Re}$ is defined as the ratio between the inertial and the viscous forces (Fig. 4)

$$
\mathrm{Re}=\mathrm{F}_{\mathrm{i}} / \mathrm{F}_{\mathrm{V}}=\left(1 / 2 \rho \pi \mathrm{r}^{2} \mathrm{v}^{2}\right) /(6 \pi \eta r v)=1 / 12(\rho \mathrm{rv} / \eta)
$$

and omitting constants

$$
\operatorname{Re}=\rho r v / \eta
$$




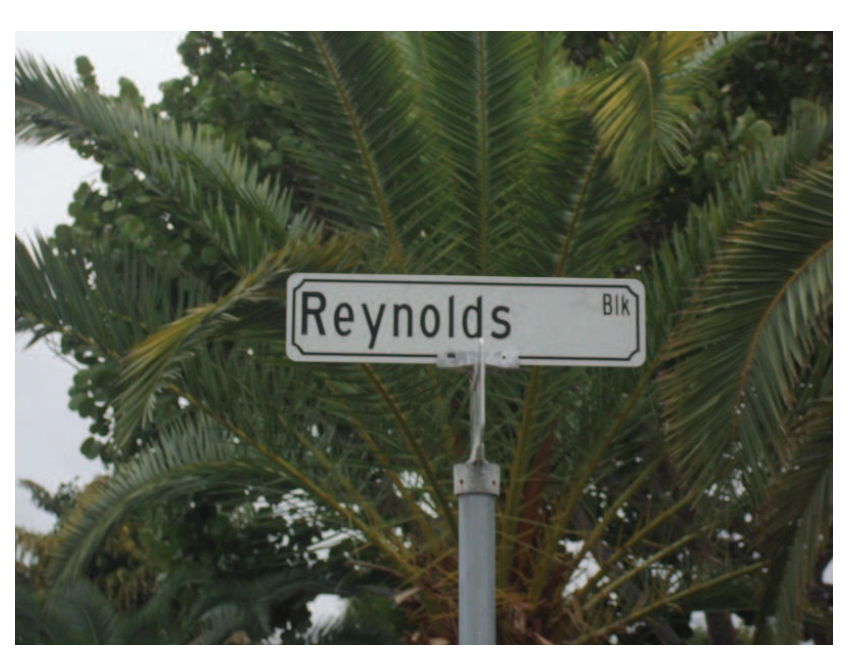

Figure 4 - Searching for the right positioning in viscous phenomena. Reynolds Street in Key West, Florida (USA).

When Re is low $(<<1)$ viscous forces prevail, as it occurs generally in tissues and in details in the ECM.

The Darcy law is an experimental constitutive equation, which describes the flow in a liquid through a porous medium and holds in a regimen of viscous flow. The preponderance of the viscous forces makes apparent a connection, generally false, between the forces which cause the movement and its velocity. It is generally true that forces are proportional to the acceleration, while in unusual viscous regimens it can resemble that they are proportional to velocity.

These same conditions are present in the electrical conduction, ruled by the $\mathrm{Ohm}$ law $\mathrm{RI}=\mathrm{V}$ or as an alternative $\mathbf{I}=\mathrm{kV}$, where $\mathbf{V}$ is the voltage, $\mathbf{I}$ the current intensity, $\mathbf{R}$ is the resistance, $\mathbf{k}$ the conductivity and the following relationship holds

$$
\mathrm{R}=\frac{1}{\mathrm{k}}
$$

The movement of electrons occurs in a viscous regimen, where a null voltage difference matches a null current intensity.

In an ideal fluid instead, according to the inertia law, movement should occur also in absence of forces or pressure gradients, but when viscous forces prevail, as occurs in the laminar flow, the Poiseuille law holds, which is a modified Ohm law with a complete specification of the resistance.

\section{The Darcy law}

The Darcy law is applied to a flow through an irregular porous medium, which experiments an additional obstacle to movement. It is an analogous of the Ohm and
Poiseuille laws and of other laws which describe a fluid movement as well as the heat propagation according to JB Fourier. The Starling law in physiology can be also derived by the Darcy law.

Referring to a specific experimental set which uses a column container and a filter (Figure Column), the Darcy law is formulated as follows

$$
\mathrm{Q}=\mathrm{kA}\left(\mathrm{h}_{1}-\mathrm{h}_{2}\right) / \mathrm{L}
$$

where $\mathbf{Q}$ is the flow, $\mathbf{k}$ is the conductivity, $\mathbf{A}$ the upstream cross section, $\mathbf{h}_{\mathbf{1}}$ the above and $\mathbf{h}_{\mathbf{2}}$ the below piezo-metric level and $\mathbf{L}$ the length of the porous filter.

Dividing by $\mathrm{A}$ and setting $\mathrm{q}=\mathrm{Q} / \mathrm{A}$ and $\nabla \mathrm{h}=\left(\mathrm{h}_{1}\right.$ $\mathrm{h}_{2}$ )/L, a generalized Ohm law is inferred.

$$
\mathrm{q}=-\mathrm{k} \nabla \mathrm{h}
$$

where $\mathrm{q}$ is the flow through a unit area and $\nabla \mathrm{h}$ (pronounced 'nabla h') is the hydrostatic pressure gradient.

It is worth noting that:

- the pressure difference is an analogous of the electrical difference of potential, thus the equivalence to the Ohm law;

- the minus sign is introduced because a mean gradient is defined as the downstream value (h2) minus the upstream value (h1), divided by the distance between the two points, i.e. just the opposite value of the terms in the first equation.

Another equivalent formulation of the Darcy law is the following

$$
\mathrm{Q}=\mathrm{kA} \frac{\mathrm{h}+\mathrm{L}}{\mathrm{L}}
$$

where $\mathrm{h}=\mathrm{h} 1-\mathrm{h} 2$ and the formula holds for vertical conduits, when the fluid gathers itself on a porous material of height $\mathrm{L}$ until a level $\mathrm{h}$, so that a global height $\mathrm{h}+\mathrm{L}$ weights on the inferior limit of the material.

The above reported formulas describe the Darcy law in static conditions, while in dynamic conditions the DarcyWeisbach formula holds, as follows

$$
\mathrm{h}_{1}=\mathrm{f} \frac{\mathrm{L}}{\mathrm{D}} \frac{\mathrm{v}^{2}}{2 \mathrm{~g}}
$$

which in pressure terms can be re-written

$$
\Delta \mathrm{p}=\mathrm{f} \frac{\mathrm{L}}{\mathrm{D}} \rho \frac{\mathrm{v}^{2}}{2}
$$

where $\Delta \mathrm{p}$ and $\mathbf{h}_{\mathbf{l}}$ are the pressure loss, $\mathbf{f}$ the Darcy friction factor, $\mathbf{L}$ the pipe length, $\mathbf{D}$ the hydraulic diameter, $\mathbf{v}$ the velocity, $\mathbf{g}$ the gravitational acceleration and $\rho$ the fluid density. 
Intuitively, the last kinetic term is reduced by a fraction equal to the friction coefficient, as many times as the length of the conduit measured in diameters.

\section{Final considerations}

As it can be easily seen, I treated essentially biophysical laws which can be applied to the ECM in lymph disease, thus defining all this as mathematics is quite inappropriate. However, I chose to maintain the original title I gave to a presentation to a Lymphedema Meeting several years $\operatorname{ago}^{8}$, in order to underline the theoretical frame, often neglected, which is at the origin of this research.

Involving the study of porous media in lymphology is not completely new in bioengineering world ${ }^{9,10}$, as the similarity was often used in the development of research models. I'm trying instead to put this research field in contact with the ultrasound clinical findings.

Circulation is commonly matched to the study of rivers in hydrography. Veins resemble the river sources which merge together into a great river, while arteries branch repeatedly, as it occurs in a river delta.

The idea of tracing back instead lymph circulation to the Darcy law makes lymphology much closer to the world of agriculture and geology, where groundwater gathers and flows following quite modified laws, in comparison with fluid dynamics or hemodynamics of big vessels.

The above similarity is not just a fascinating image, as it also offers the opportunity of using in lymphology several tools which are commonly used in geology.

\section{Conclusions}

The extracellular matrix is an important compensating pathway in lymph diseases, when lymph vessels are partially or totally incapable to drain tissues. The ultrasound examination show several frequent modifications, constituted by tissue granularity and wide interposed spaces. Flow is mainly viscous and obeys to the Darcy law, commonly used to model flow in porous media. This theoretical frame interprets lymphology as a discipline which is close to agriculture and geology. An interesting suggestion is then to adopt in lymphology several tools in use in geological studies.

\section{Acknowledgements}

I thank Mrs. Iolanda Palma for her invaluable help in typesetting the manuscript.

\section{Endnotes}

[1] Henry Darcy is also well know for having perfectioned the previous and only theoretical Pitot tube, producing a sophisticated tool for the daily use of engineers and professionals in hydrogeology. He would never imagine that his invention would have an additional wide use in a future discipline like aeronautics, being then part of the essential instruments in every aircraft (in another fluid, air and not water). He could also be considered a model of clear scientific behaviour and ethics, as he chose to neglect any patent for his invention, giving it instead to the scientific community. He had no opportunity however to see the great developments of his research, as he died while he was still perfectioning the instrument. I report a few lines from the obituary notes written by Charié-Marsaines (1858), as translated from French by Brown (2003):

"Perfections brought by Darcy to this instrument are considerable and he would have been able to take a patent to profit from its exclusive manufacture during a certain number of years. However, as a believer in the customs of disinterestedness, he decided to abandon his invention to the public. The administration has already made a number of these instruments some of which are placed in the precision instrument deposit at l'École des Ponts et Chaussées, and others sent to state engineers to be used in operations that demand exact measurements of the water velocity."

\section{References}

1) Albergati FG, Bacci PA, Mancini S. La matrice extracellulare. Minelli Ed, Arezzo 2004. ISBN: 88-9011405-0-X.

2) Passariello F, Carbone, R, Mancini, A. Semeiotica ultrasuonografica delle alterazioni linfatiche nell'arto inferiore. Min. Angiol. $1991 ; 16$ :suppl. 1 al n. 2, 453-7. Translation and reprint. [Ultrasound Semeiotics of the Lymphatic Modifications of the Lower Limbs]. JTAVR. 2016 Nov;1(2):131-135. DOI: 10.24019/jtavr.17.

3) Wikipedia. [Internet]. Henry Darcy. Available on line a the address https://en.wikipedia.org/wiki/Henry Darcy at the date Mar 8, 2017.

4) Darcy H. The public fountains of the city of Dijon. Paris. Victor Dalmont, 1856.

5) Brown GO. 2003. Henry Darcy's perfection of the Pitot tube, in Henry P. G. Darcy and Other Pioneers in Hydraulics: Contributions in Celebration of the 200th Birthday of Henry Philibert Gaspard Darcy, Brown GO, Garbrecht JD, and Hager WH (eds), ASCE, Reston, VA. pg. 14-23.

6) Charié-Marsaines PG. (1858). [French] "Notice necrologique sur M. Darcy, Inspecteur Général des Ponts et Chaussées", Annales des Ponts et Chaussées. 1858; Series 3; 15, 90-109. Available online at the address http://gallica.bnf.fr/ark:/12148/bpt6k408489d.image.f93 at the date of Mar 8, 2017.

7) Darcy H. [French] Note relative à quelques modifications à introduire dans le tube de Pitot. Annales des Ponts et Chaussées. 1858; Series 3; 15, 351-9. Available online at the address http://gallica.bnf.fr/ ark:/12148/bpt6k408489d.image.f354 at the date of Mar 8, 2017.

8) Passariello F. La Matematica del Linfedema. [Mathematics of Lymphedema]. VIII theoretical and practical workshop. Diagnosis and treatment of (phlebo)lymphedema. Apr $23^{\text {rd }}, 2006$, Bologna, Italy.

9) Swartz MA, Kaipainen A, Netti PA, Brekken C, Boucher Y, Grodzinsky AJ, Jain RK. Mechanics of interstitial-lymphatic fluid transport: theoretical foundation and experimental validation. Journal of Biomechanics 1999 May; 32:1297-307.

10) Swartz MA. The physiology of the lymphatic system. Advanced Drug Delivery Review. 2001;50:3-20. 